\title{
Numerical Simulation of Macrosegregation Caused by Thermal-Solutal Convection and Solidification Shrinkage Using ALE Model
}

\author{
Kang-Xin Chen ${ }^{1} \cdot$ Hou-Fa Shen ${ }^{1}$ \\ Received: 5 December 2018 / Revised: 19 March 2019 / Published online: 16 April 2019 \\ (c) The Chinese Society for Metals (CSM) and Springer-Verlag GmbH Germany, part of Springer Nature 2019
}

\begin{abstract}
Solidification shrinkage has been recognized as an important factor for macrosegregation formation. An arbitrary Lagrangian-Eulerian (ALE) model is constructed to predict the macrosegregation caused by thermal-solutal convection and solidification shrinkage. A novel mesh update algorithm is developed to account for the domain change induced by solidification shrinkage. The velocity-pressure coupling between the non-homogenous mass conservation equation and momentum equation is addressed by a modified pressure correction method. The governing equations are solved by the streamline-upwind/ Petrov-Galerkin-stabilized finite element algorithm. The application of the model to the $\mathrm{Pb}-19.2 \mathrm{wt} \% \mathrm{Sn}$ alloy solidification problem is considered. The inverse segregation is successfully predicted, and reasonable agreement with the literature results is obtained. Thus, the ALE model is established to be a highly effective tool for predicting the macrosegregation caused by solidification shrinkage and thermal-solutal convection. Finally, the effect of solidification shrinkage is analyzed. The results demonstrate that solidification shrinkage delays the advance of the solidification front and intensifies the segregation.
\end{abstract}

Keywords Macrosegregation $\cdot$ Solidification shrinkage $\cdot$ Finite element method $\cdot$ Arbitrary Lagrangian-Eulerian (ALE)

\section{Introduction}

During solidification of metal alloys, large-scale non-uniformity in local solute composition (i.e., macrosegregation) is likely to arise. The defect causes a wide variation in the properties of the casting and impairs the performance of the final components. Macrosegregation results from the relative motion between the solute-rich liquid phase and solute-depleted solid phase [1]. The main factors influencing the relative motion are the thermal-solutal buoyancy forces, solidification shrinkage, grain sedimentation and solid deformation [2].

Modeling and simulation have been widely used to investigate macrosegregation during the past decades. Up to the

Available online at http://link.springer.com/journal/40195

Hou-Fa Shen

shen@tsinghua.edu.cn

1 Key Laboratory for Advanced Materials Processing Technology, Ministry of Education, School of Materials Science and Engineering, Tsinghua University, Beijing 100084, China present, substantial progress has been made in the development of macrosegregation models; among these, the singledomain models based on the classical mixture theory [3] or volume-averaging method [4] are the most popular. In numerous numerical studies, the solidification shrinkage is omitted in the single-domain models by using equal densities for the solid and liquid phases. During solidification, the volume shrinkage in the mushy zone is compensated by the melt in the risers, inducing a feeding flow in the liquid and mushy zones. Although the shrinkage-induced flow may be weak, it is likely to strongly impact the formation of macrosegregation [5].

One of the earliest attempts to consider solidification shrinkage in the single-domain models was by Chiang and Tsai [6]. They modified the continuum model developed by Bennon and Incropera and considered the domain change by using the front tracking method. The model was used to analyze the interaction between shrinkage-induced flow and natural convection for solidifying alloys in a two-dimensional rectangular cavity with a riser. Diao and Tsai [7] later extended the model to include the solute equation and studied the combined effect of solidification shrinkage, natural convection and change in the cross section on the formation 
of macrosegregation. Krane and Incropera [8] examined the effect of solidification shrinkage on the macrosegregation formation in a Pb-19.2 wt\% Sn alloy which was cooled at a side wall based on a modified continuum model. With regard to the treatment of solidification shrinkage, an inlet velocity boundary condition was provided on a portion of the top surface; moreover, the feeding velocity was calculated from the volume required to compensate the shrinkage. They clarified that the solidification shrinkage flow was impelled by mass conservation requirements throughout the domain rather than the viscous stress terms in the momentum equation. Heinrich and Poirier [9] examined the effect of solidification contraction during directional solidification of hypoeutectic $\mathrm{Pb}-\mathrm{Sn}$ alloys based on the volume-averaging model; they successfully predicted the inverse segregation at the cooled surface. Samanta and Zabaras [10] studied the convection and macrosegregation impelled by buoyancy and shrinkage for solidifying a Pb-19.2 wt\% Sn alloy by using stabilized finite element techniques.

In the above studies, small test cases were considered, and certain simplifications of the free surface and boundary conditions were carried out. An inflow boundary condition on a part of the upper boundary or a riser with a flat moving free surface was imposed to feed the shrinkage. However, these assumptions are not valid for steel ingot castings, where a shrinkage pipe forms during solidification. Thus, to predict the macrosegregation formation and solidification shrinkage pipe simultaneously, the free surface evolution has to be solved while predicting segregation formation.

Zhang et al. [11] developed a single-domain multiphase model for macrosegregation and shrinkage pipe formation and utilized a volume of fluid (VOF) method to track the free surface evolution. Wang et al. [12, 13] and Wu et al. [14, 15] extended the traditional multiphase models by including an additional gas phase to treat the formation of the shrinkage cavity and investigated the interaction between the shrinkage cavity and macrosegregation. In these studies, fixed mesh methods were adopted, in which the location of the free surface was tracked through a variable defined on the mesh such as the VOF method or the gas-phase-included multiphase model method. The moving mesh method, or arbitrary Lagrangian-Eulerian (ALE) method, can also be used to solve the free surface problem. The advantage of this method lies in the natural representation of the free surfaces, which yields an accurate description of the front. Bellet and coworkers introduced this method into the solidification and casting context in order to simulate the mold filling process and shrinkage cavity formation $[16,17]$. However, the utilization of the ALE method to develop macrosegregation models for considering the effect of solidification shrinkage has been rarely reported.

In this study, an ALE model is developed to predict macrosegregation caused by the combined effect of solidification shrinkage and thermal-solutal convection during solidification of metal alloys. First, the governing equations of the modified continuum model are deduced based on the ALE theory. Secondly, an ALE-based finite element algorithm is developed for solving the coupled governing equations including the mesh update algorithm and the modified pressure correction method. Finally, the ALE model is utilized to study the solidification progress of a $\mathrm{Pb}-19.2 \mathrm{wt} \% \mathrm{Sn}$ alloy and the effect of solidification shrinkage is analyzed.

\section{Mathematical Model}

The mathematical model used in this study is modified from the continuum model developed by $\mathrm{Ni}$ and Incropera [18]. The conservation equations for mass, momentum, energy and solute are developed based on the following assumptions:

1. The liquid is Newtonian and incompressible, and the flow is laminar.

2. The solid phase is stationary; thus, neither grain sedimentation nor mushy zone deformation is considered.

3. The mushy zone is modeled as an isotropic porous medium saturated with liquid, and the permeability is defined by the Carman-Kozeny formula.

4. The densities of the solid and liquid phases are different albeit constant, and the Boussinesq approximation is used in the buoyancy term of the momentum conservation equation.

5. The thermal-physical properties of the solid and liquid phases are different albeit constant.

6. The level rule is used to describe microsegregation.

7. Neither porosity nor internal shrinkage is considered during solidification; thus, no gas phase is present in the casting.

The governing equations of the modified continuum model are commonly derived in the Euler framework based on the mixture theory. In this study, to handle the domain change caused by solidification shrinkage, an ALE technique is utilized. ALE formulations are adapted from the Eulerian formulations, as expressed by Eqs. (1)-(4). Details about the ALE adaption are available in the literature [19].

Mass conservation:

$\frac{\partial \rho(\chi, t)}{\partial t}+\left(\boldsymbol{u}(\chi, t)-\boldsymbol{u}_{\mathrm{mesh}}(\chi, t)\right) \cdot \nabla \rho(\chi, t)+\rho(\chi, t) \nabla \cdot \boldsymbol{u}(\chi, t)=0$. 
Momentum conservation:

$$
\begin{array}{r}
\rho(\chi, t) \frac{\partial \boldsymbol{u}(\chi, t)}{\partial t}+\rho(\chi, t)\left(\boldsymbol{u}(\chi, t)-\boldsymbol{u}_{\mathrm{mesh}}(\chi, t)\right) \cdot \nabla \boldsymbol{u}(\chi, t)=\nabla \cdot\left(\mu_{1} \frac{\rho(\chi, t)}{\rho_{1}} \nabla \boldsymbol{u}(\chi, t)\right) \\
-\nabla P(\chi, t)-\frac{\mu_{1}}{K} \frac{\rho(\chi, t)}{\rho_{1}} \boldsymbol{u}(\chi, t)+\rho(\chi, t) \boldsymbol{g}\left(\beta_{\mathrm{T}}\left(T(\chi, t)-T_{\mathrm{ref}}\right)+\beta_{\mathrm{w}}\left(w_{1}(\chi, t)-w_{\text {ref }}\right)\right) .
\end{array}
$$

Energy conservation:

$$
\begin{gathered}
\rho(\chi, t) \frac{\partial H(\chi, t)}{\partial t}-\rho(\chi, t) \boldsymbol{u}_{\mathrm{mesh}}(\chi, t) \cdot \nabla H(\chi, t)+\rho(\chi, t) \boldsymbol{u}(\chi, t) \cdot \nabla H_{1}(\chi, t) \\
=\nabla \cdot(k(\chi, t) \nabla T(\chi, t))+\left(H(\chi, t)-H_{1}(\chi, t)\right) \nabla \cdot(\rho(\chi, t) \boldsymbol{u}(\chi, t)) .
\end{gathered}
$$

Solute conservation:

$$
\begin{gathered}
\rho(\chi, t) \frac{\partial w(\chi, t)}{\partial t}-\rho(\chi, t) \boldsymbol{u}_{\mathrm{mesh}}(\chi, t) \cdot \nabla w(\chi, t)+\rho(\chi, t) \boldsymbol{u}(\chi, t) \cdot \nabla w_{1}(\chi, t) \\
=\nabla \cdot\left(\rho(\chi, t) f_{1} D_{1} \nabla w_{1}(\chi, t)\right)+\left(w(\chi, t)-w_{1}(\chi, t)\right) \nabla \cdot(\rho(\chi, t) \boldsymbol{u}(\chi, t)) .
\end{gathered} .
$$

In the above equations, $\chi$ denotes the ALE spatial system, $t$ is the time, $\rho$ is the mixture density, $\boldsymbol{u}$ is the mixture velocity, $\boldsymbol{u}_{\text {mesh }}$ is the mesh velocity, $\mu_{1}$ is the dynamic viscosity of the liquid phase, $P$ is the pressure, $K$ is the permeability, $\rho_{1}$ is the liquid density, $\boldsymbol{g}$ is the gravity acceleration, $\beta_{\mathrm{T}}\left(\beta_{\mathrm{w}}\right)$ is the thermal (solutal) expansion coefficient, $T$ is the temperature, $T_{\text {ref }}$ is the reference temperature, $w_{\text {ref }}$ is the reference mass fraction of the solute, $H$ is the mixture enthalpy, $H_{1}$ is the liquid enthalpy, $k$ is the mixture thermal conductivity, $w$ is the mixture solute mass fraction of solute, $w_{1}$ is the mass fraction of the solute in liquid phase, $f_{1}$ is the liquid mass fraction and $D_{1}$ is the diffusion coefficient in liquid.

Because the densities of the solid and liquid phases are unequal, the mass fraction and volume fraction are different. Thus, it is necessary to distinguish between the two types of fractions in the variable definition. The mixture density and mixture thermal conductivity are volume-averaged; these are expressed as

$\rho=\rho_{\mathrm{s}} g_{\mathrm{s}}+\rho_{1} g_{1}$

$k=k_{\mathrm{s}} g_{\mathrm{s}}+k_{1} g_{1}$.

The mixture enthalpy and mixture solute mass fraction are mass-averaged; these are expressed as

$H=f_{1} H_{1}+f_{\mathrm{s}} H_{\mathrm{s}}$,

$w=f_{1} w_{1}+f_{\mathrm{s}} w_{\mathrm{s}}$.

The permeability is a function of the volume fractions; it is defined as

$K=\lambda_{2}^{2} g_{1}^{3} / g_{\mathrm{s}}^{2} / 180$,

where $\lambda_{2}$ is the secondary dendrite arm spacing. In Eqs. (5)-(9), $f$ denotes the mass fraction; $g$ denotes the volume fraction; and the subscript s or 1 denotes the solid or liquid phase, respectively.

In the ALE formulations, the variables are defined in the ALE system and the mesh is updated during each time step to account for the solidification shrinkage

$X(\chi, t+\Delta t)=X(\chi, t)+\boldsymbol{u}_{\text {mesh }}(\chi, t) \cdot \Delta t$,

where $X$ denotes the global vector of nodal coordinates. The mesh velocity, $\boldsymbol{u}_{\text {mesh }}$, is determined by the mesh update algorithm, which will be introduced in the next section.

\section{Numerical Method}

\subsection{Mesh Update Algorithm}

During solidification of castings, shrinkage cavities form at the riser. The formation process of the shrinkage cavities corresponds to the evolution of the free surface. In this section, the ALE mesh update algorithm for tracking the free surface evolution during the formation of shrinkage cavity is introduced.

The simplified assumptions are as follows: First, it is assumed that the fluctuations of the free surface are negligible (and thus omitted); moreover, only the decline in the liquid level owing to the solidification shrinkage is considered. Secondly, for the nodes at the free surface, if the solid fraction is larger than the critical solid fraction, $g_{\text {sc }}$, these nodes stay stationary. For the other part of the free surface, which is generally at the middle of the free surface, the surface tension is omitted, and the melt is assumed to flow down with the feeding velocity. 
The following objectives should be achieved by the ALE mesh update algorithm. First, the domain change of the casting caused by solidification shrinkage should be described by the updated mesh. Therefore, the boundary nodes at the free surface are supposed to track the evolution of the free surface owing to solidification shrinkage. Secondly, the positions of the internal nodes should be adjusted by a suitable algorithm to reserve adequate mesh quality. The update algorithms for the boundary nodes and the internal nodes are introduced as follows:

\section{Boundary nodes}

To determine the feeding velocity for the boundary nodes, the solidification shrinkage should be calculated. First, the total shrinkage volume is calculated. The solidification shrinkage is defined by the source term of the mass conservation equation as follows:

$S_{\rho}=\frac{1}{\rho(\chi, t)}\left(\frac{\partial \rho(\chi, t)}{\partial t}+\left(\boldsymbol{u}(\chi, t)-\boldsymbol{u}_{\mathrm{mesh}}(\chi, t)\right) \cdot \nabla \rho(\chi, t)\right)$.

Thus, the total shrinkage volume can be computed as the volumetric integral of the source term:

$V_{\mathrm{shr}}=\int_{V} S_{\rho} \mathrm{d} \Omega$.

Next, the shrinkage area of the free surface is calculated. The shrinkage function is defined as follows:

$\delta=\left\{\begin{array}{ll}1, & \text { if } g_{\mathrm{s}}<g_{\mathrm{sc}} \\ 0, & \text { if } g_{\mathrm{s}}>g_{\mathrm{sc}}\end{array}\right.$.

The shrinkage area of the free surface can be computed as the surface integral of the shrinkage function:

$S_{\mathrm{shr}}=\int_{S} \delta \mathrm{d} \Gamma$

Finally, the shrinkage displacement and the mesh node velocity at the free surface are calculated as follows:

$d_{\mathrm{shr}}=V_{\mathrm{shr}} / S_{\mathrm{shr}}$,

2. Interior nodes

$U_{\text {mesh }}=d_{\mathrm{shr}} / \Delta t \cdot \delta$.

Compared with the boundary nodes, which should track the free surface, the movements of the interior nodes are largely arbitrary. However, the new positions of the mesh nodes should be placed to maintain adequate mesh quality so that no re-meshing is required owing to excessive mesh distortion. There are numerous mesh update algorithms. The Laplacian smoothing method is used in this study; it is based on updating the positions of the nodes by solving the Laplace equation.
Details about the Laplacian smoothing method are available in Ref. [19].

\subsection{Solution Algorithm}

The mathematical model adopted in this study is deduced in the ALE framework to consider the domain change owing to the solidification shrinkage of castings. The model covers the coupling among the fluid flow, the heat transfer and solute transport during solidification, and the update of the mesh. The finite element method is adopted to solve the coupled conservation equations of mass, momentum, energy and solute. For solving the coupled mass and momentum equations-Eqs. (1) and (2), a modified pressure correction method is adopted as follows:

Tentative velocity step:

$$
\begin{aligned}
& \rho \frac{\boldsymbol{u}^{*}-\boldsymbol{u}^{n-1}}{\Delta t}+\rho\left(\boldsymbol{u}^{n-1}-\boldsymbol{u}_{\mathrm{mesh}}\right) \cdot \nabla \boldsymbol{u}^{*} \\
& =\nabla \cdot \mu_{1} \frac{\rho}{\rho_{1}} \nabla \boldsymbol{u}^{*}-\frac{\mu_{1}}{K} \frac{\rho}{\rho_{1}} \boldsymbol{u}^{*}-\nabla P^{n-1} \\
& \quad+\rho \boldsymbol{g}\left(\beta_{\mathrm{T}}\left(T-T_{0}\right)+\beta_{\mathrm{C}}\left(w_{1}-w_{1, \mathrm{ref}}\right)\right) .
\end{aligned}
$$

Pressure correction step:

$\nabla \cdot \frac{1}{\rho} \nabla\left(p^{n}-p^{*}\right)=\frac{1}{\Delta t}\left(\frac{1}{\rho}\left(\frac{\partial \rho}{\partial t}+\left(\boldsymbol{u}^{n-1}-\boldsymbol{u}_{\mathrm{mesh}}\right) \cdot \nabla \rho\right)+\nabla \cdot \boldsymbol{u}^{*}\right)$.

Velocity update step:

$\frac{\boldsymbol{u}^{n}-\boldsymbol{u}^{*}}{\Delta t}=-\frac{1}{\rho} \nabla\left(P^{n}-P^{*}\right)$.

The streamline-upwind/Petrov-Galerkin (SUPG) method is utilized to stabilize the convection terms in the transport equations for momentum, energy and solute. For computing the energy and solute conservation equations, the nonlinearity induced by the temperature-solute coupling during solidification is addressed by the Newton-Rapson method.

Because the model covers the multiphysics coupling and mesh update, a segregated method is adopted as follows:

1. Initialize the variables at the current time step with those at the previous time step.

2. Solve the conservation equations of mass and momentum based on the modified stabilized pressure correction method.

3. Solve the nonlinear energy conservation equation with the Newton-Rapson method.

4. Solve the nonlinear solute conservation equation with the Newton-Rapson method. 

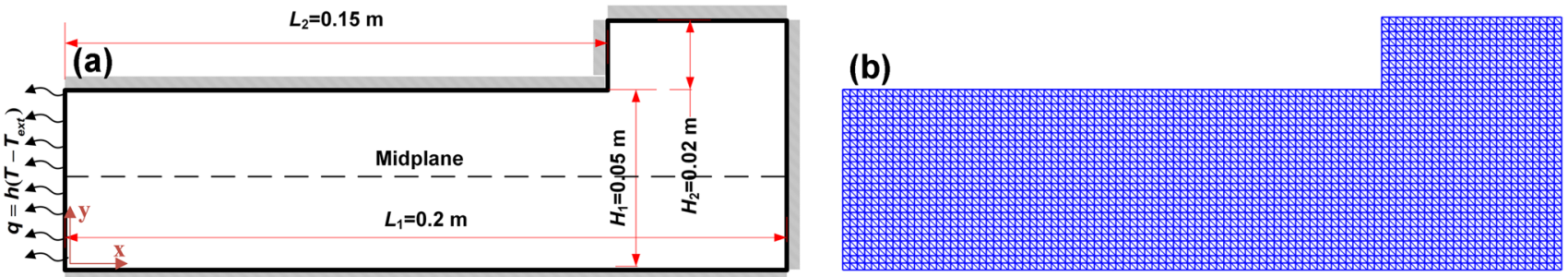

Fig. 1 a Schematic, b structured mesh for $\mathrm{Pb}-19.2 \mathrm{wt} \% \mathrm{Sn}$ solidification problem

Table 1 Thermal-physical properties and computational parameters used in calculations

\begin{tabular}{lll}
\hline Properties & Units & Value \\
\hline Liquid density, $\rho_{\mathrm{l}}$ & $\mathrm{kg} \mathrm{m}^{-3}$ & 10,000 \\
Solid density, $\rho_{\mathrm{s}}$ & $\mathrm{kg} \mathrm{m}^{-3}$ & 10,800 \\
Specific heat in liquid, $c_{\mathrm{l}}$ & $\mathrm{J} \mathrm{kg}^{-1} \mathrm{~K}^{-1}$ & 154.7 \\
Specific heat in solid, $c_{\mathrm{s}}$ & $\mathrm{J} \mathrm{kg}^{-1} \mathrm{~K}^{-1}$ & 177.9 \\
Thermal conductivity in liquid, $k_{\mathrm{l}}$ & $\mathrm{W} \mathrm{m}^{-1} \mathrm{~K}^{-1}$ & 22.9 \\
Thermal conductivity in solid, $k_{\mathrm{s}}$ & $\mathrm{W} \mathrm{m}^{-1} \mathrm{~K}^{-1}$ & 39.7 \\
Latent heat, $L$ & $\mathrm{~J} \mathrm{~kg}^{-1}$ & 30,162 \\
Liquid dynamic viscosity, $\mu_{1}$ & $\mathrm{~kg} \mathrm{~m}^{-1} \mathrm{~s}^{-1}$ & $2.3 \times 10^{-3}$ \\
Thermal expansion coefficient, $\beta_{\mathrm{T}}$ & $\mathrm{K}^{-1}$ & $1.09 \times 10^{-4}$ \\
Solutal expansion coefficient, $\beta_{\mathrm{w}}$ & $(\mathrm{wt} \%)^{-1}$ & $3.54 \times 10^{-3}$ \\
Secondary dendrite arm spacing, $\lambda_{2}$ & $\mathrm{~m}$ & $71 \times 10^{-6}$ \\
Melting point of the pure metal, $T_{\mathrm{f}}$ & ${ }^{\circ} \mathrm{C}$ & 327.5 \\
Liquidus slope, $m$ & $\mathrm{~K}(\mathrm{wt} \%)^{-1}$ & -2.334 \\
Partition coefficient, $k_{\mathrm{P}}$ & & 0.31 \\
Eutectic temperature, $T_{\text {eut }}$ & ${ }^{\circ} \mathrm{C}$ & 183.0 \\
Eutectic composition, $w_{\text {eut }}$ & $\mathrm{wt} \%$ & 61.9 \\
Nominal concentration, $w_{0}$ & $\mathrm{wt} \%$ & 19.2 \\
Initial temperature, $T_{0}$ & ${ }^{\circ} \mathrm{C}$ & 287 \\
Heat transfer coefficient & $\mathrm{W} \mathrm{m}^{-2} \mathrm{~K}^{-1}$ & 1000 \\
External temperature & ${ }^{\circ} \mathrm{C}$ & 20 \\
Diffusion coefficient in liquid, $D_{1}$ & $\mathrm{~m} \mathrm{~m}^{2} \mathrm{~s} \mathrm{~s}^{-1}$ & $1.05 \times 10^{-9}$ \\
\hline
\end{tabular}

5. Calculate the difference in velocity, temperature and solute concentration between two adjacent iterations. If they are not converged, return to step (2).
6. Calculate the solidification shrinkage, update the mesh based on the ALE algorithm and update the mesh velocity.

7. Advance time to the next time step.

In the above segregated method, the mesh is updated only one time at each time step, whereas multiple iterations are performed for coupling the conservation equations to achieve synchronization among the coupling fields.

\section{Results and Discussion}

To test and validate the developed ALE macrosegregation model, a Pb-19.2 wt\%Sn casting, which was addressed earlier [10], is considered. The problem involves the solidification of a $\mathrm{Pb}-19.2 \mathrm{wt} \% \mathrm{Sn}$ alloy in a square cavity with a hot top. The square cavity is of length $0.2 \mathrm{~m}$ and height $0.05 \mathrm{~m}$; moreover, the hot top of length $0.05 \mathrm{~m}$ and height $0.02 \mathrm{~m}$ is located on the upper right side of the cavity. The domain is thermally insulated on all the surfaces except the left lateral side, which is subject to convective cooling condition; the heat transfer coefficient is $1000 \mathrm{~W} /\left(\mathrm{m}^{2} \mathrm{~K}\right)$. Nonslip boundary conditions are assumed. The computation domain with boundary conditions and the structured triangular mesh used in the calculation are shown in Fig. 1. The thermal-physical properties and computational parameters are presented in Table $1[10]$. (a)

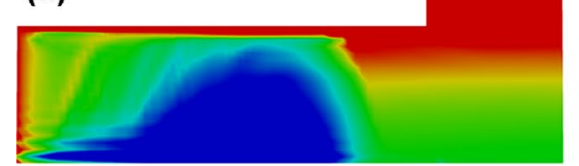

(b)

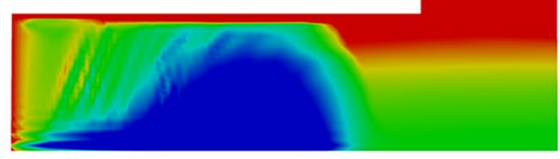

$\begin{array}{lllll}0.202 & 0.197 & 0.192 & 0.187 & 0.182\end{array}$

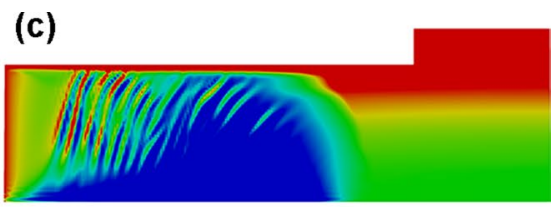

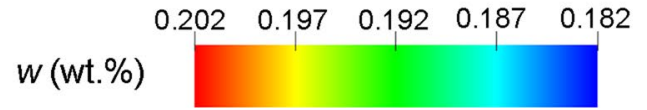

Fig. 2 Distributions of tin concentration at time $t=400 \mathrm{~s}$ predicted using different meshes: a Mesh I, b Mesh II, c Mesh III 
Table 2 Comparison of maximum and minimum concentrations in midplane of casting for the three meshes

\begin{tabular}{|c|c|c|c|c|c|c|c|c|}
\hline \multirow{2}{*}{$\begin{array}{l}\text { Solidifica- } \\
\text { tion time (s) }\end{array}$} & \multicolumn{4}{|c|}{$w_{\max }(\mathrm{wt} \%)$} & \multicolumn{4}{|c|}{$w_{\min }(\mathrm{wt} \%)$} \\
\hline & Mesh I & Mesh II & Mesh III & $\operatorname{Ref}[10]$ & Mesh I & Mesh II & Mesh III & $\operatorname{Ref}[10]$ \\
\hline 50 & 20.27 & 20.26 & 20.27 & 20.0 & 18.86 & 18.61 & 17.83 & 18.8 \\
\hline 100 & 20.27 & 20.26 & 20.27 & 20.1 & 18.62 & 18.48 & 17.66 & 18.6 \\
\hline 200 & 20.27 & 20.26 & 20.50 & 20.2 & 18.36 & 18.26 & 17.74 & 18.3 \\
\hline 400 & 20.27 & 20.26 & 20.73 & 20.2 & 17.90 & 17.88 & 17.48 & 17.4 \\
\hline
\end{tabular}

\subsection{Validation and Mesh Convergence Study}

In this section, a mesh convergence study is conducted with three triangular structured meshes: Mesh I, Mesh II, and Mesh III. Their mesh sizes are $2.5 \mathrm{~mm}, 2.0 \mathrm{~mm}$, and $1.0 \mathrm{~mm}$, respectively.

Figure 2 shows the distribution of the solute concentration at time $t=400 \mathrm{~s}$. The solute concentration distributions predicted by using the three meshes are similar, except that the channel segregation features are more developed for Mesh III. The formation of the channel segregation is related to the flow perturbation or instability in the mushy zone. When the thermal-solutal convection is adequately strong, flow instability occurs; this in turn destabilizes the mushy zone and causes the channel segregation [20].

To quantitatively study the effect of mesh size on the macrosegregation formation, the comparison of the maximum and minimum concentrations in midplane at different times for the three meshes is presented in Table 2. Predictions by using a similar mesh as Mesh I are reported in Ref [10]; these are included in Table 2 for validation.

First, comparing the predictions by using Mesh I with those in Ref [10], a moderate agreement is obtained; thus, it can validate the ALE solidification model and the finite element algorithm. Secondly, the concentrations predicted by using Mesh I and Mesh II are similar to each other, whereas the macrosegregation predictions by using Mesh III at times $t=200 \mathrm{~s}$ and $t=400 \mathrm{~s}$ are substantially severer than those by using Mesh I and Mesh II, as shown in Table 2. This difference results from the significant channel segregation features predicted by using Mesh III.

\subsection{Solidification Progress and Solidification Shrinkage Effect}

In order to investigate the effect of solidification shrinkage on macrosegregation, two cases are set up. For case 1, both solidification shrinkage and thermal-solutal convection are considered; moreover, the complete ALE model is utilized. For case 2, solidification shrinkage is omitted by assuming equal densities for the solid and liquid phases. In case 2 , the computation domain does not change during solidification, and the ALE model degenerates to the classical fixed-domain continuum model [3] without solidification shrinkage. In the following part of this section, the results calculated for the two cases are compared and analyzed to investigate the effect of solidification shrinkage. Mesh II is used in both the cases.

The solidification progress of the $\mathrm{Pb}-19.2 \mathrm{wt} \% \mathrm{Sn}$ alloy is characterized by the advance of the mushy zone and the evolution of fluid flow in the mushy and liquid zones (Fig. 3). Figure 3a-d corresponds to the ALE model considering both the thermal-solutal buoyancy flow and shrinkage-induced flow. Figure $3 \mathrm{e}-\mathrm{h}$ corresponds to the simplified model considering only the thermal-solutal buoyancy force. The alloy has a wide freezing range of approximately $100{ }^{\circ} \mathrm{C}$. Thus, a large mushy zone can be observed, as indicated by the color map of the solid volume fraction and the white contour lines of the solid fraction in Fig. 3. As the solidification proceeds, the mushy zone advances from left to right gradually. Because the Sn-enriched interdendritic melt has a lower density than that of the bulk melt, the solutal convection dominates over the opposing thermal convection $[8,9]$. Thus, a large counterclockwise circulation forms, as shown in Fig. 3a. Moreover, the height of the hot top predicted by ALE model decreases with time owing to solidification shrinkage, as shown in Fig. 3a-d. An apparent reduction in the hot top height can also be observed at time $t=1000 \mathrm{~s}$ by comparing Fig. 3d, h. In this study, because the hot top is located at a distance from the chill wall of the cavity, the melt in the hot top solidifies last and the liquid level is flat. This coincides with the liquid level assumption used in Ref [10].

Although the solidification progress predicted by the ALE shrinkage model is highly similar to that by the simplified model without shrinkage, certain differences are apparent; this is evident by comparing Fig. 3a-d, e-h.

First, the white contour lines of the solid volume fraction in Fig. 3a-d are less forward than those in Fig. 3e-h. That is, if solidification shrinkage is considered, the advance of the mushy zone is marginally delayed compared with the case without shrinkage. The reason is apparent; when solidification shrinkage is considered, the hot melt is induced to flow into the mushy zone, and then the advance of the mushy zone is delayed. However, this does not imply that the consideration of solidification shrinkage will extend the predicted solidification time. On the contrary, owing to the 

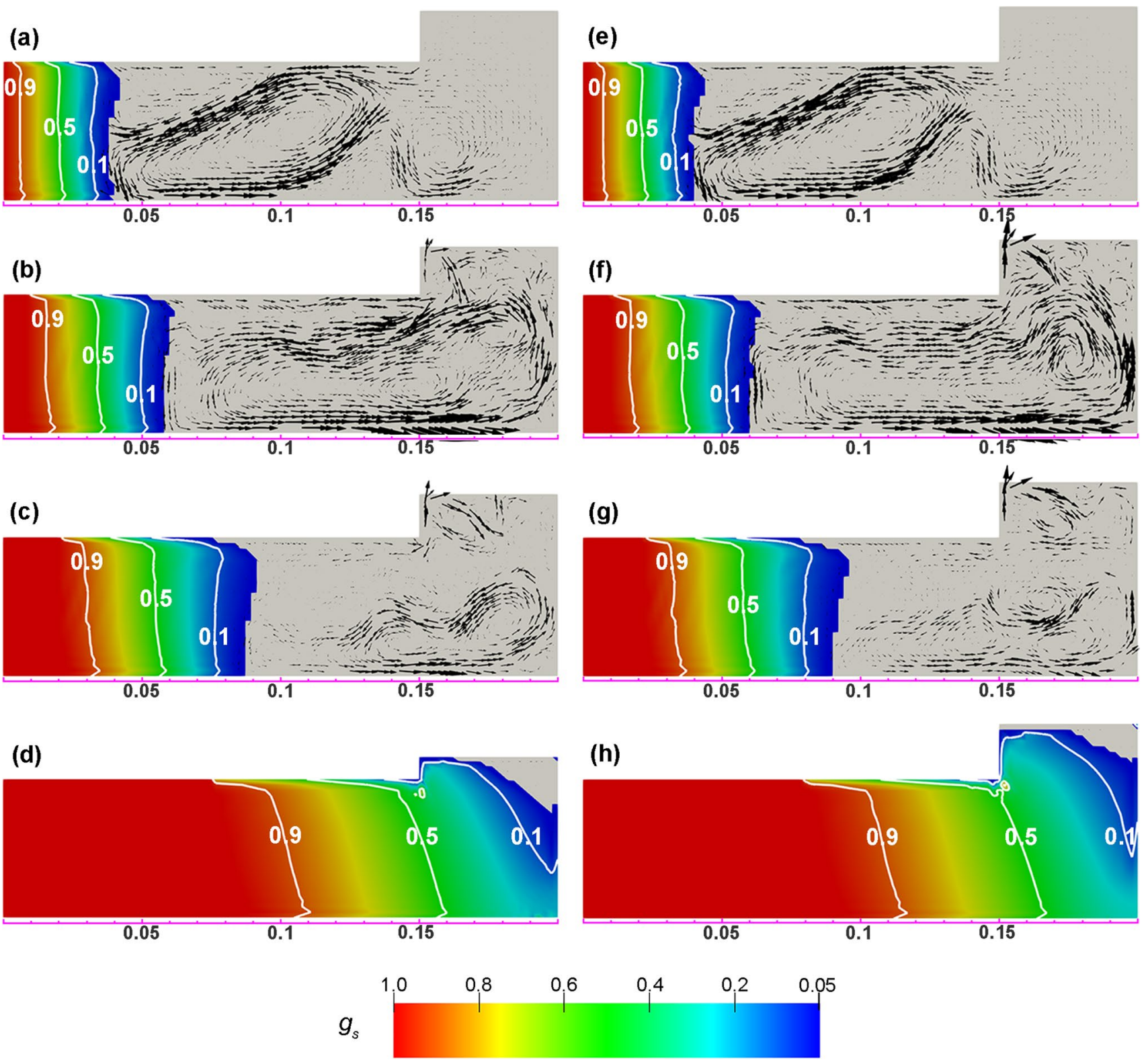

Fig. 3 Solidification sequence and fluid flow of Pb-19.2 wt\%Sn alloy at different times a-d correspond to the ALE shrinkage model, $\mathbf{e}-\mathbf{h}$ correspond to the simplified model without shrinkage: a, e $50 \mathrm{~s}, \mathbf{b}, \mathbf{f} 100 \mathrm{~s}, \mathbf{c}, \mathbf{g} 200 \mathrm{~s}, \mathbf{d}, \mathbf{h} 1000 \mathrm{~s}$

reduced volume of casting, the total solidification time predicted with the ALE shrinkage model is less than that with the model without shrinkage.

Secondly, the difference in the velocity field at time $t=50 \mathrm{~s}$ is negligible, as shown in Fig. 3a, e. However, at times $t=100 \mathrm{~s}$ and $t=200 \mathrm{~s}$, difference in the distribution of the velocity vector between the two cases is apparent (Fig. 3b, c, f, g). This can be explained as follows: The flow is induced by the combined effect of thermal-solutal buoyancy and solidification shrinkage in Fig. 3a-c. At the beginning of the solidification process, the thermal-solutal buoyancy effect is significantly larger compared with the solidification shrinkage effect. Therefore, the influence of solidification shrinkage on the fluid flow is negligible. As solidification proceeds, the flow weakens owing to the increase in the solidification fraction. Then, the influence of the solidification shrinkage gradually becomes significant. Thus, it can be concluded that the solidification shrinkage exerts an apparent impact on the fluid flow at the subsequent stage of solidification (when the thermal-solutal flow weakens). 
(a)

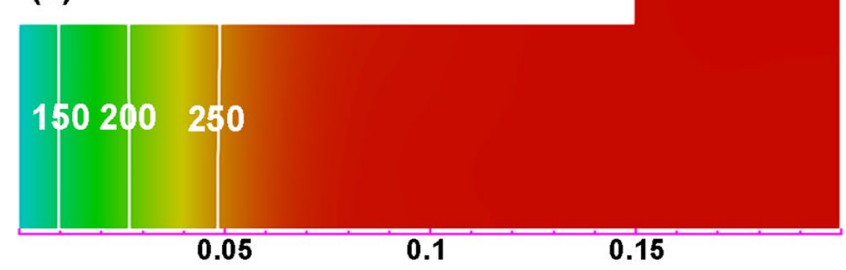

(b)

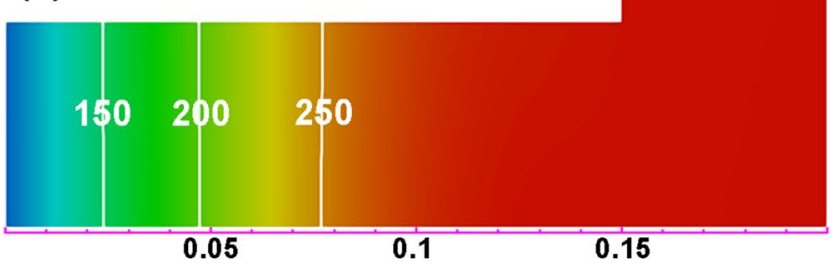

0.05

(c)

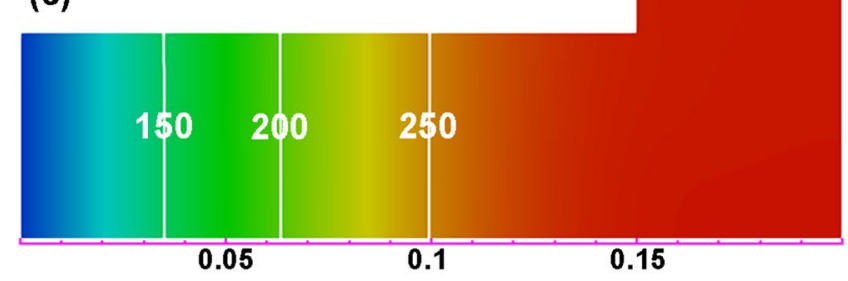

(d)

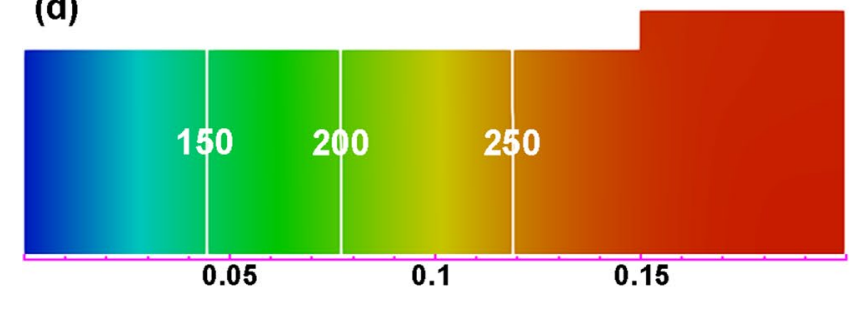

(e)

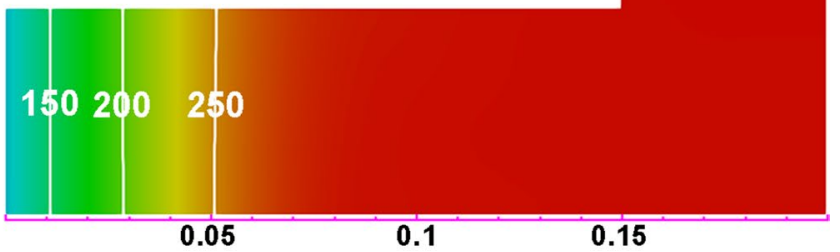

(f)

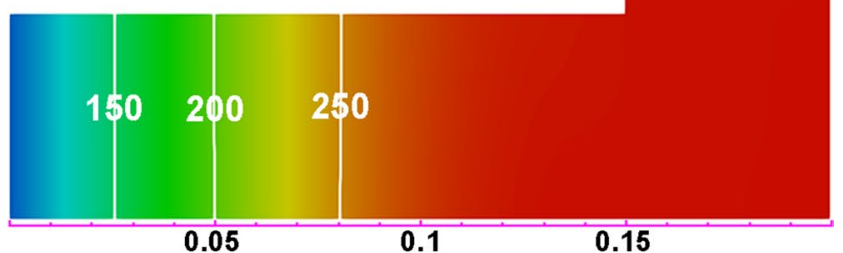

(g)

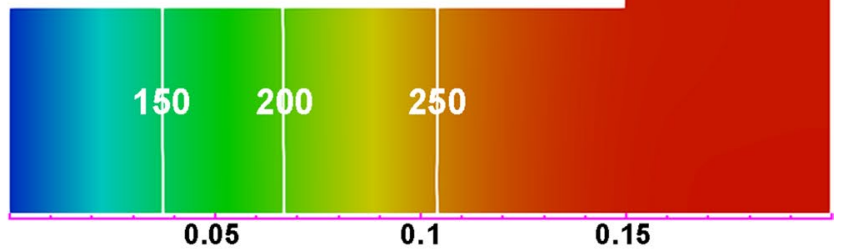

(h)

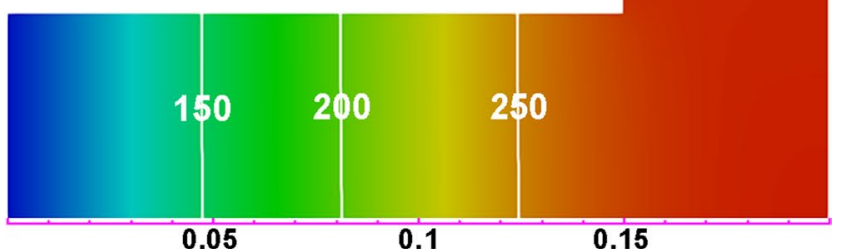

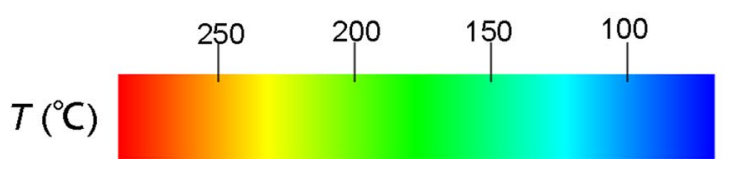

Fig. 4 Evolution of temperature field of $\mathrm{Pb}-19.2 \% \mathrm{Sn}$ alloy at different times $\mathbf{a}-\mathbf{d}$ corresponds to the ALE shrinkage model, e-h corresponds to the simplified model without shrinkage: a, e $200 \mathrm{~s}, \mathbf{b}, \mathbf{f} 400 \mathrm{~s}, \mathbf{c}, \mathbf{g} 600 \mathrm{~s}, \mathbf{d}, \mathbf{h} 800 \mathrm{~s}$

Figure 4 shows the evolution of the temperature field during solidification. Figure $4 \mathrm{a}-\mathrm{d}$ corresponds to the ALE model, and Fig. 4e-h corresponds to the model without shrinkage. Although the temperature field predicted by the ALE shrinkage model is highly similar to that by the simplified model without shrinkage, the white contour lines of the temperature field in Fig. 4a-d are less forward than those in Fig. 4e-h. This also results from the delay caused by the solidification shrinkage. The temperature evolutions are also presented in Ref [10]; however, the delay caused by solidification shrinkage was not indicated.

Figure 5 shows the evolution of the Sn concentration distribution. Figure 5a-d corresponds to the ALE model, and
Fig. 5e-h corresponds to the model without shrinkage. The solidification progress is indicated by the white contour lines of the solid volume fraction. For both cases, a thin positive segregation layer forms near the top surface of the cavity; moreover, negative segregation forms from the left at the bottom of the cavity, as shown in Fig. 5. It should be noted that positive segregation is predicted at the left chill wall by the ALE model, as shown in Fig. 5a-d. This is a typical inverse segregation caused by solidification shrinkage [1, 2]; this will be analyzed further below. However, no apparent positive segregation at the left chill wall is predicted in Fig. 5e-h. Whereas inverse segregation has been commonly observed at the chill wall of castings $[6,7]$, the prediction 
(a)

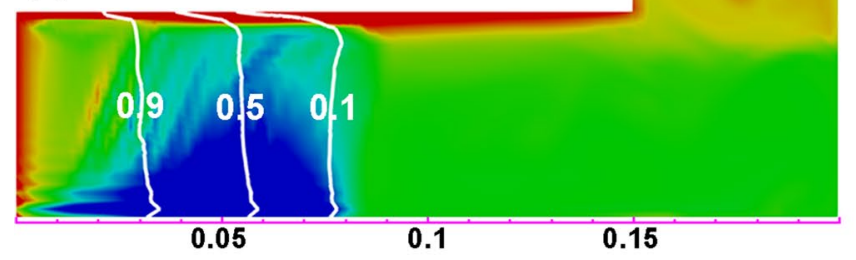

(b)

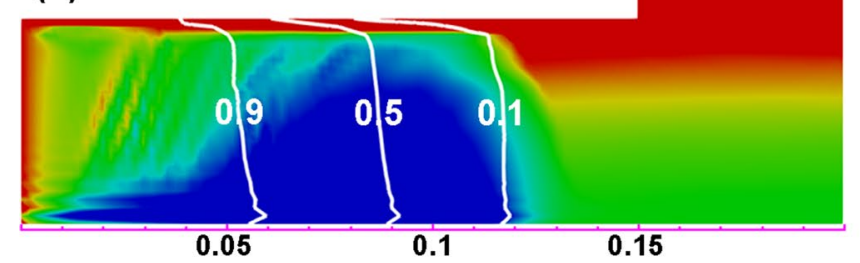

0.05

(c)

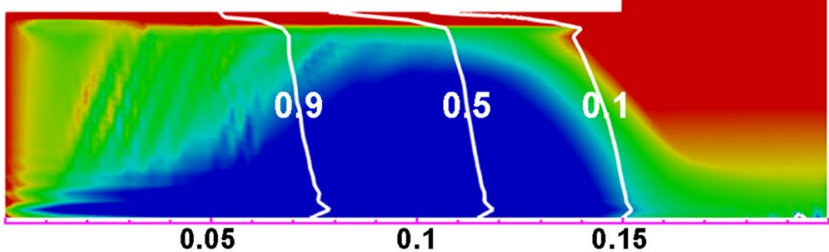

(d)

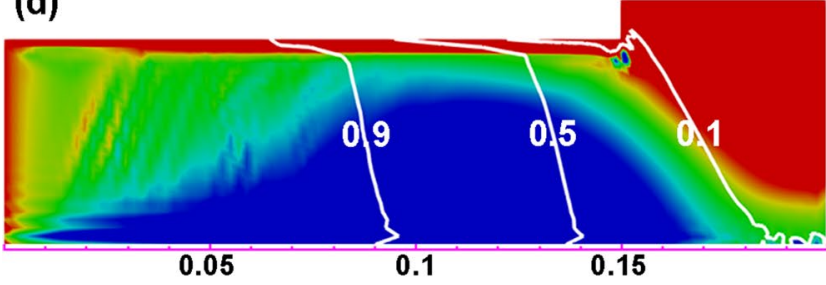

(e)

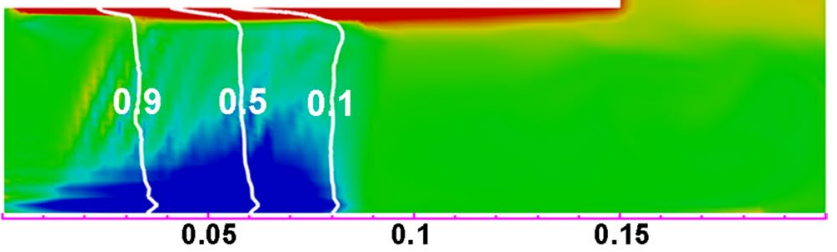

(f)

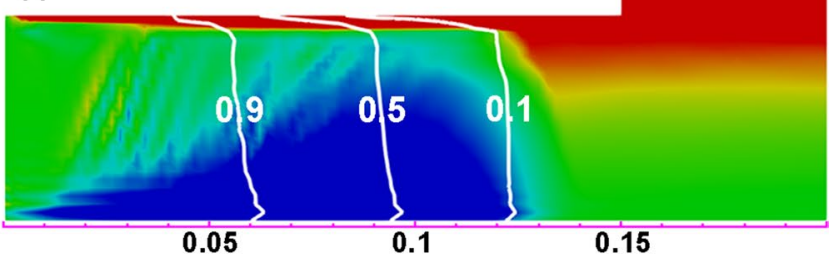

(g)

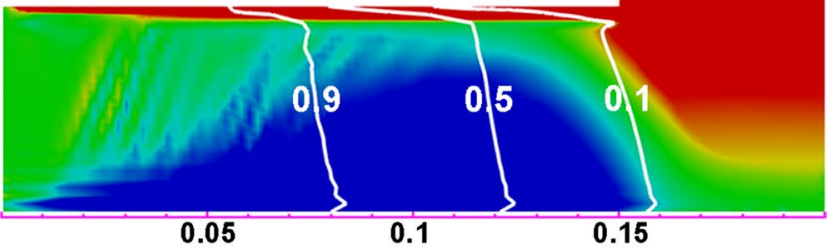

(h)

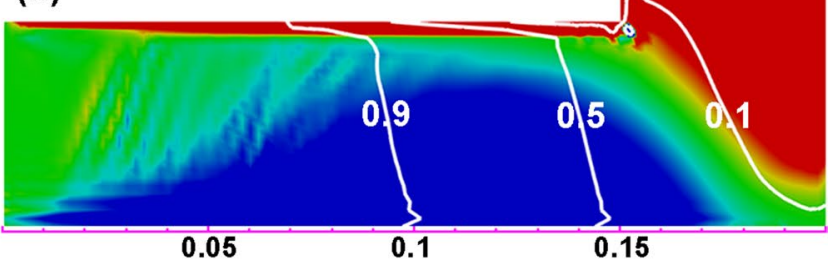

$0.187 \quad 0.182$

$w(w t . \%)$

Fig. 5 Evolution of Sn concentration distribution of $\mathrm{Pb}-19.2 \% \mathrm{Sn}$ alloy at different times $\mathbf{a}-\mathbf{d}$ corresponds to the ALE shrinkage model, $\mathbf{e}-\mathbf{h}$ corresponds to the simplified model without shrinkage: a, e $200 \mathrm{~s}, \mathbf{b}, \mathbf{f} 400 \mathrm{~s}, \mathbf{c}, \mathbf{g} 600 \mathrm{~s}, \mathbf{d}, \mathbf{h} 800 \mathrm{~s}$

of this phenomenon relies on the proper consideration of solidification shrinkage in the macrosegregation model.

Figure 6 represents the evolution of the solute concentration profiles along the mid-height of the casting. In each subfigure, predictions for both cases with and without solidification shrinkage are plotted. For the case with solidification shrinkage, the formation process of inverse segregation at the left chill wall is apparent in Fig. 6. However, when shrinkage is omitted, there is no apparent segregation at the left chill wall. Moreover, the fluctuations on the profiles that represent the channel segregation are predicted for both the cases. Furthermore, the segregation degrees of the negative and positive segregation regions predicted by the ALE model are marginally severer than those by the model without solidification shrinkage.

To better understand the formation mechanism of macrosegregation and the effect of solidification shrinkage, the finite element form of the solute conservation equation is analyzed as follows. The weak form of the solute conservation equation, Eq. (4), can be simplified as 


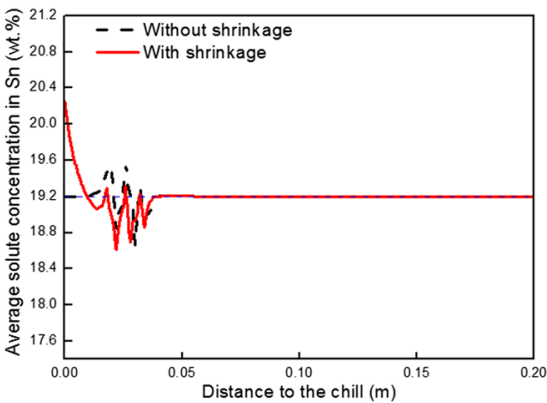

(a)

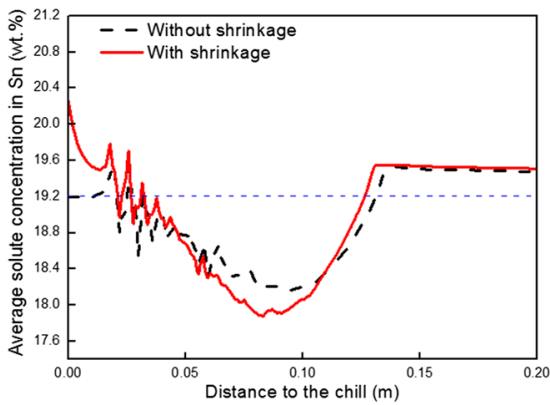

(d)

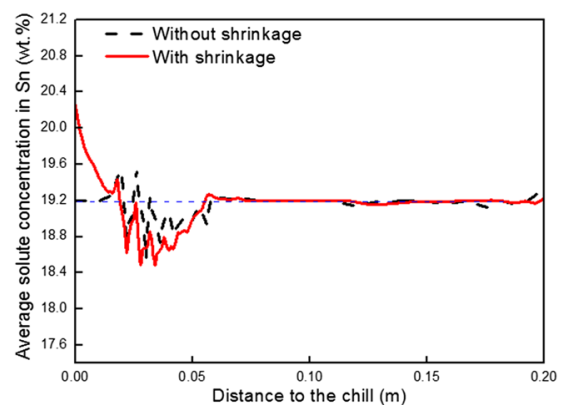

(b)

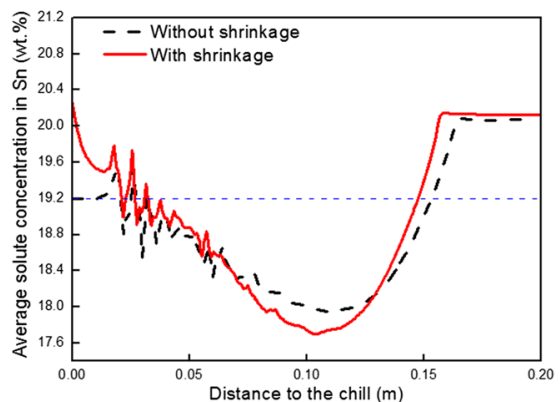

(e)

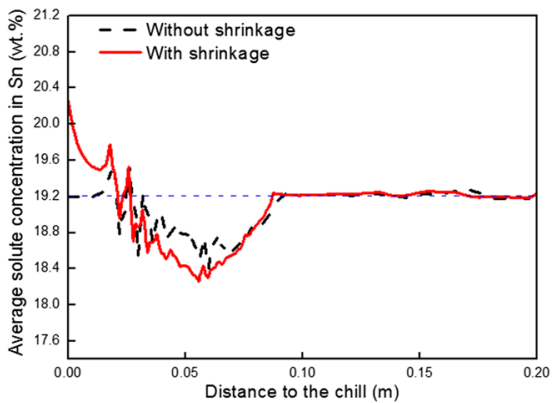

(c)

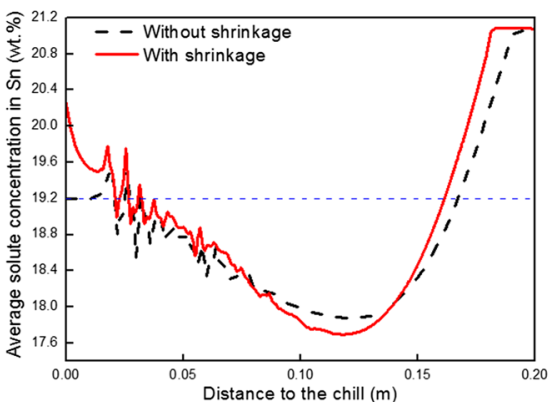

(f)

Fig. 6 Concentration of tin in mid-height of casting at different times: a 50 s, b 100 s, c 200 s, d 400 s, e 600 s, f 800 s

$$
\begin{aligned}
& \int N_{\mathrm{i}} \frac{\partial w}{\partial t} \mathrm{~d} \Omega=\int_{\text {diffusion boundary integral }} N_{\mathrm{i}} f_{1} D_{1} \nabla w_{1} \cdot \boldsymbol{n} \mathrm{d} \Gamma-\int f_{1} D_{1} \nabla w_{1} \cdot \nabla N_{\mathrm{i}} \mathrm{d} \Omega-\int N_{\text {diffusion }} \boldsymbol{u} \cdot \nabla w_{1} \mathrm{~d} \Omega
\end{aligned}
$$

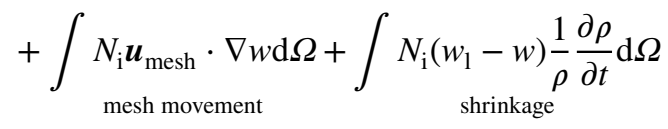

where $N_{\mathrm{i}}$ denotes the test function, $\Omega$ denotes the domain and $\boldsymbol{n}$ is the unit vector normal to the domain boundary $\Gamma$. The left-side term represents the change rate of the solute concentration, and the right-side terms function as a solute redistribution function [9]. The different contributions to the formation of macrosegregation can be clearly identified: diffusion boundary integral, diffusion in liquid phase and transport owing to the thermal-solutal convection, ALE mesh movement and solidification shrinkage. For the model without solidification shrinkage, the mesh movement term and shrinkage term vanish.

The formation of inverse segregation is analyzed as follows: The diffusion boundary integral is retained here to eliminate the false flux on the boundary [9]. Owing to the very low diffusion coefficient $D_{1}$, of the order of $10^{-9} \mathrm{~m}^{2} \mathrm{~s}^{-1}$, the contributions of the diffusion boundary and diffusion in liquid are negligible. Moreover, the velocity near the leftside wall is essentially perpendicular to the temperature gradient; thus, the contribution of the advection term is also rather limited. Therefore, when no solidification shrinkage is considered, the change rate of the solute concentration at the left cool wall is very low; no apparent segregation is observed near the left-side wall in Fig. 6. For the ALE model considering solidification shrinkage, the mesh movement term and shrinkage term exist. The contribution of the mesh movement term is limited because the nodes at the left side do not shift significantly. The solidification shrinkage term is positive because the liquid concentration is generally larger than the average concentration, and the density increases during solidification [11]. Therefore, the shrinkage term is the cause of the positive segregation or the inverse segregation at the left cool side of the casting.

\section{Conclusions}

In this study, a novel ALE-based finite element model is developed to predict the macrosegregation induced by solidification shrinkage and thermal-solutal convection of binary alloys. The governing equations for the conservation 
of mass, momentum, energy and species are derived in the ALE framework and solved by stabilized finite element techniques. A new mesh update algorithm based on Laplacian smoothing is developed to consider volume shrinkage. For the coupling between the non-homogenous mass conservation equation and momentum equation, a modified pressure correction method is developed. The SUPG method is adopted to stabilize the convection terms in the conservation equations of momentum, energy and species. The ALE solidification model is applied to a Pb-19.2 wt\%Sn alloy solidification problem. The results are in good agreement with those in the literature results, demonstrating the applicability and accuracy of the model. The inverse segregation is successfully predicted by using the ALE solidification model. Furthermore, the effect of solidification shrinkage is investigated. The conclusions are as follows:

1. For the melt flow, solidification shrinkage apparently impacts the subsequent stage of solidification (when the thermal-solutal convection flow turns weak).

2. The advance of the solidification front is delayed by the hot feeding flow when considering solidification shrinkage.

3. With regard to the macrosegregation, when considering solidification shrinkage, the inverse segregation is predicted at the cool surface of casting; moreover, the degrees of segregation at both the negative and positive segregation regions are enhanced by the solidification shrinkage.

Acknowledgements This work was supported by the National Natural Science Foundation of China-Liaoning Joint Fund (U1508215).

\section{References}

[1] M.C. Flemings, ISIJ Int. 40, 833-841 (2000)

[2] C. Beckermann, Int. Mater. Rev. 47, 243-261 (2002)

[3] W.D. Bennon, F.P. Incropera, Int. J. Heat Mass Transf. 30, 21612170 (1987)

[4] C. Beckermann, R. Viskanta, PhysicoChem. Hydrodyn. 10, 195213 (1988)

[5] X. Ma, D. Li, ISIJ Int. 54, 356-358 (2014)

[6] K.C. Chiang, H.L. Tsai, Int. J. Heat Mass Transf. 35, 1763-1770 (1992)

[7] Q.Z. Diao, H.L. Tsai, Metall. Mater. Trans. A 25, 1051-1062 (1994)

[8] M.J.M. Krane, F.P. Incropera, Metall. Mater. Trans. A 26, 2329 2339 (1995)

[9] J.C. Heinrich, D.R. Poirier, Model. Simul. Mater. Sci. 12, 881-899 (2004)

[10] D. Samanta, N. Zabaras, Int. J. Numer. Methods Eng. 64, 17691799 (2005)

[11] S. Zhang, J. Yanke, D.R. Johnson, M.J.M. Krane, Int. J. Numer. Methods Heat 24, 468-482 (2014)

[12] T.M. Wang, S. Yao, X.G. Zhang, J.Z. Jin, M. Wu, A. Ludwig, Acta Metall. Sin. 42, 584-590 (2006)

[13] T.M. Wang, T.J. Li, Z.Q. Cao, J.Z. Jin, T. Grimmig, A. BuhrigPolaczek, Acta Metall. Sin. 42, 591-598 (2006)

[14] M. Wu, A. Kharicha, A. Ludwig, IOP Conference Series: Materials Science and Engineering, vol 84, p. 12006 (2015)

[15] M. Wu, A. Ludwig, A. Kharicha, Appl. Math. Model. 41, 102-120 (2017)

[16] M. Bellet, V.D. Fachinotti, Comput. Methods Appl. Mech 193, 4355-4381 (2004)

[17] M. Bellet, O. Jaouen, I. Poitrault, Int. J. Numer. Methods Heat Fluid Flow 15, 120-142 (2005)

[18] J. Ni, F.P. Incropera, Int. J. Heat Mass Transf. 38, 1271-1284 (1995)

[19] T. Belytschko, W.K. Liu, B. Moran, Nonlinear Finite Elements for Continua and Structures, 2nd edn. (Wiley, Hoboken, 2014), pp. 417-475

[20] Y.F. Cao, Y. Chen, D.Z. Li, H.W. Liu, P.X. Fu, Metall. Mater. Trans. A 47, 2927-2939 (2016) 